\title{
A Review of Patient Preferences for Osteoporosis Drug Treatment
}

\author{
Mickaël Hiligsmann ${ }^{1}$ • Sandrine P. G. Bours ${ }^{2}$ - Annelies Boonen ${ }^{2}$
}

Published online: 19 August 2015

(C) The Author(s) 2015. This article is published with open access at Springerlink.com

\begin{abstract}
Poor medication adherence is a major problem in chronic diseases such as osteoporosis that may partially be due to unaddressed patient values and preferences. Data on patient preferences could help clinicians to improve medication adherence and could also be useful in policy decisions and guideline development. This paper aims to identify literature reporting on the preferences of patients for osteoporosis drug medications. Several methods have been used to elicit patient preferences for medications and their characteristics including qualitative research, survey with ranking/rating exercises, discrete-choice experiments and clinical studies (crossover designs, open-label study). All these studies revealed that osteoporotic patients have preferences for medications and their attributes, in particular for less-frequent dosing regimens. Interestingly, variations in the preferences of patients were observed in most studies, suggesting the importance to take into account individual preference in decision-making to improve osteoporosis care.
\end{abstract}

This article is part of the Topical Collection on Health Economics and Quality of Life

Mickaël Hiligsmann

m.hiligsmann@maastrichtuniversity.nl

Sandrine P. G. Bours

s.bours@mumc.nl

Annelies Boonen

a.boonen@mumc.nl

1 Department of Health Services Research, School for Public Health and Primary Care (CAPHRI), Maastricht University, P.O. Box 616, 6200 MD Maastricht, The Netherlands

2 Department of Internal Medicine, CAPHRI, Maastricht University Medical Centre, Maastricht, The Netherlands
Keywords Crossover designs - Discrete-choice experiment Drug therapy $\cdot$ Osteoporosis $\cdot$ Preferences $\cdot$ Review ·

Satisfaction

\section{Introduction}

Osteoporosis represents an increasing public health problem, especially in the Western world. For the year 2010, it was estimated that about 27.5 million of people from the European Union have osteoporosis [1], resulting in approximately 3.5 million new fractures. Despite the fact that several drugs have demonstrated to be safe and effective in reducing the risk of fractures [2], adherence to medications remains poor and suboptimal [3], with substantial clinical and economic implications [4]. Poor adherence to therapy may partially be due to unaddressed patient values and preferences [5].

Understanding what patients prefer and involving them in clinical decision-making could lead to improved satisfaction with therapy and hence medication adherence [6]. Patient perceptions and preferences with osteoporosis medications were shown to impact adherence behaviour $[7 \cdot \bullet]$ and discontinuation rates [8]. The patient's perspective is now becoming increasingly important in the design and assessment of healthcare interventions [9]. Patients want to be informed by their doctors and play an active role in clinical decisionmaking [10]. Therefore, in recent years, there has been a growing interest in studies to elicit preferences for healthcare interventions. There are different ways to elicit patient preferences including qualitative research, survey with ranking/ rating exercises, discrete-choice experiments and clinical studies (such as crossover or open-label study).

With the development of new osteoporosis medications that differ mainly according to mode of administration, it is not surprising that several studies have been conducted to 
elicit patient preferences for osteoporosis medications and understand the so-called attributes or factors that contribute to preferences. Reviewing these studies and reporting their findings could have substantial potential for clinicians towards improving poor medication adherence and could also be very useful for policy decisions and guideline development [11]. This paper was therefore designed to review studies that assessed preferences in osteoporosis. A secondary aim was to discuss how preference could be incorporated in clinical decision-making.

\section{Patients' Preferences for Osteoporosis Medications}

A review of published studies that describe patient preferences for osteoporosis drug treatment was conducted in PubMed. The search terms were 'preference* [title/ abstract] OR satisfaction [title/abstract] AND 'osteoporosis [MESH term]', and all articles published until May 1, 2015, were included. We only included original research that looks at preferences for osteoporosis medications and/or medication attributes. We followed the operational definition of preference given by Joy et al. [11]: 'given a choice, the selection of an alternative'. We therefore included studies that looked at the choice of patient between alternatives. We also included preference studies that assessed preferences for medication attributes using hypothetical treatment options and studies that were interested in preferences for medication characteristics. Non-medication interventions were excluded. Abstract and title screening was initially performed, followed by a full-text screening. References of identified articles were searched for additional articles and completed by authors' knowledge of the published literature. Data were collected on study authors, country, publication year, method, sample size, funding and main findings. The number of medication attributes was included for surveys, and interventions were included for clinical studies.

The PubMed search yielded 246 articles. A total of 23 articles met our inclusion criteria. Ten studies used a questionnaire or interviews to elicit preferences for medication attributes. Among these, 4 studies specifically aimed to identify the attributes that play a role to preferences of osteoporotic patients for medication attributes, 4 used conjoint analysis methods to elicit the trade-offs patients make between attributes and 2 were interview-based surveys to elicit preferences between two medications. The remaining 13 articles investigated patient preferences between two real-life interventions using an open-label study $(n=7)$ or crossover design $(n=6)$. Tables 1 and 2 summarize the characteristics and main findings of all these studies.

\section{Importance of Medication Attributes}

Four studies assessed the importance of osteoporosis medication attributes $[12,13,14 \bullet, 15 \bullet]$. The PREFER study was a large-scale survey that was conducted in the USA [12] and in five other countries [13]. Participants from these countries were administered an online questionnaire in which they were asked to rate and rank the importance of seven (or eight) medication attributes in determining their preferences for osteoporosis medications. In Hiligsmann et al. [14•], patient group discussions were conducted to prioritize a list of 12 potentially important medication attributes identified from the literature and discussion with clinicians. A total of five focus groups were conducted in the Netherlands and Belgium. Silverman et al. [15•] asked participants to evaluate the relative weight of specific statements using MaxDiff analyses. In MaxDiff analyses, participants receive a list of statements and are asked to indicate the most (and the least) important one.

As shown in Table 1, in all these studies, drug effectiveness was the most important attribute for the patients followed by side effects. Out-of-pocket costs and mode/frequency of administration were also considered important. Variations in the preferences of patients were reported. Hiligsmann et al. [14•] revealed different findings across the focus groups. The country system could also influence the preferences. In the Netherlands, where patients have no out-of-pocket contribution, the cost attribute was not as important as in Belgium [14•]. Silverman et al. [15•] also reported a significant impact of age, income, education and prior fractures on overall ranking but not of racial/ethnic differences.

\section{Conjoint Analyses Studies}

Four studies used conjoint analysis methods to assess trade-off that patients make between medication attributes [16-18, 19••]. Fraenkel et al. [18] used 'adaptive conjoint analyses' to determine the treatment preferences for oral bisphosphonates by frequency of administration. Results suggested that preferences are strongly influenced by route of administration. Three discrete-choice experiments (DCEs) were conducted $[16,17,19 \bullet \bullet$. A DCE is a form of conjoint analysis that describes an intervention by its attributes (e.g. effectiveness, side effects, costs) and reports how patient's preference for an intervention is influenced by the type and levels of these attributes [20]. In the DCEs, patients were asked to choose between two unlabelled drug treatments (A and B) and sometimes a third 'no treatment' option. De Bekker-Grob et al. [17] evaluated the preferences of patients for osteoporosis treatments in the Netherlands. All attributes (i.e. effectiveness, side effects, treatment duration, route of drug administration, and out-of-pocket costs) were shown to be important. The timing of administration was however limited to a maximum of 4 months. New therapies with longer 


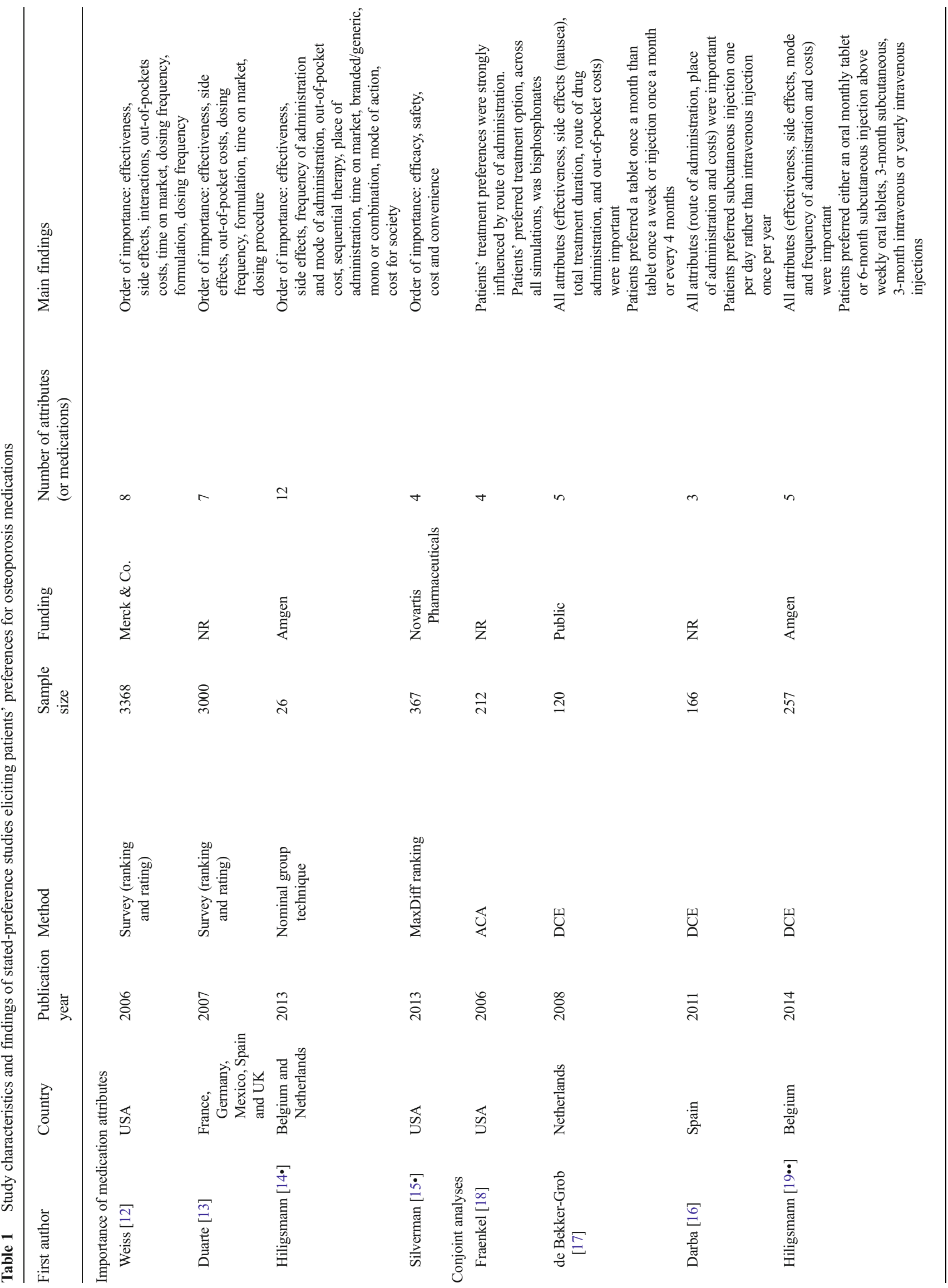




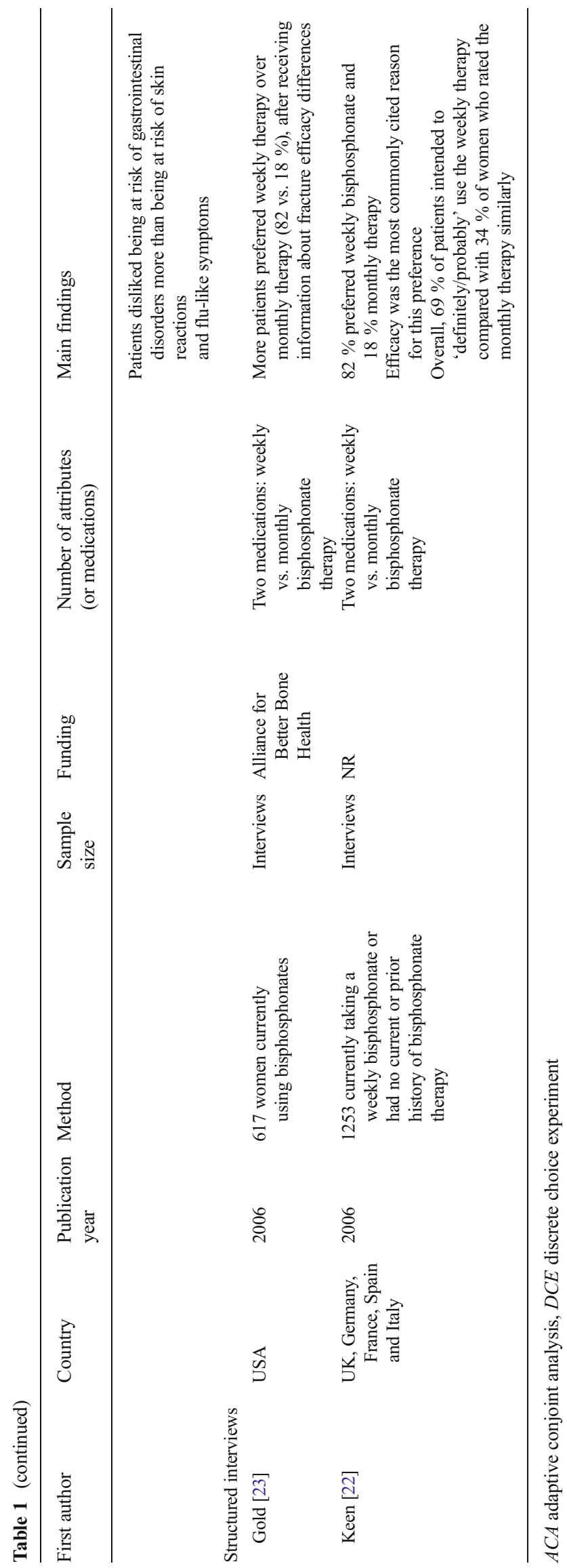

dosing intervals have recently become available, and preferences for these new administration schemes should be investigated. Darba et al. (2010) also used a DCE to investigate the importance of different treatment aspects in Spain. Only three attributes were however included in the experimental design (i.e. type of drug administration, place of administration, cost) and were all important. More recently, Hiligsmann et al. [19••] conducted a DCE with Belgian patients. This study confirms expected results that patients prefer treatments offering higher efficacy, lower costs and less-frequent dosing regimens. This study does, in addition, evaluate additional administration routes and the side effects deemed important by patients [21•]. Patients had a preference for a 6-month subcutaneous injection and weekly oral tablet compared with oral tablet and yearly intravenous. Using a mixed logit model that allows coefficients to vary between patients, this study also revealed that preferences could substantially differ between patients.

\section{Structured Interviews}

There were 2 studies conducting structured interviews by phone [22] or from a panel of patients currently using oral bisphosphonates [23]. In Keen et al. [22], 50 \% of the participants of the interviews previously or currently used oral bisphosphonates. Participants were asked to choose between two oral treatments, a weekly oral tablet with proven efficacy at the spine and hip and a monthly oral tablet with no proven efficacy at the hip. Most participants chose the treatment with proven efficacy at both hip and spine, even though the other choice was the less frequently dosed treatment [22, 23]. Previous bisphosphonate use did not influence preference; however, it affected 'intention to use' significantly in a positive direction [22].

\section{Clinical Studies}

Most preference studies identified in this review were clinical studies where patients were asked to describe their preferences and satisfaction to treatment that they had received or tested. Most studies have focused on the influence of dosing frequency (daily, weekly, monthly, bi-annually or annually) for therapy on patient preference. Studies were either crossover or open-label studies.

In the clinical studies, the following questionnaires were used: Beliefs about Medicines Questionnaire (BMQ, including 22 questions in the following major domains: the necessity of the prescribed medication to manage osteoporosis now and in the future, concerns about the potential adverse effects of taking the prescribed medication, preference for one medication over the other) [24, 25], Preference Satisfaction Questionnaire (PSQ, measuring preference, pill satisfaction, injection satisfaction, pill bother and injection bother) [25-28], questionnaires for preference adapted from Balto I and II [29-31], 


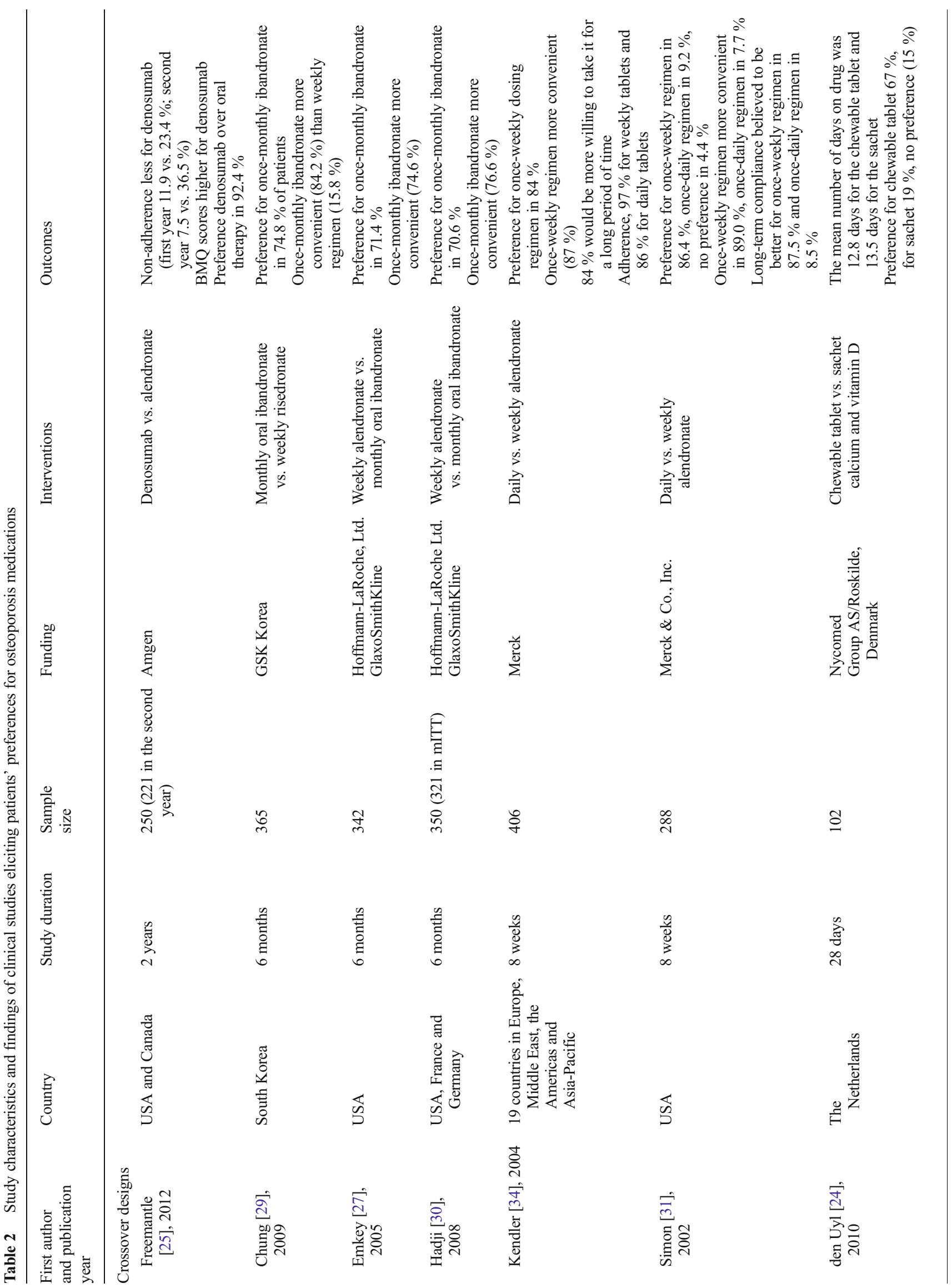




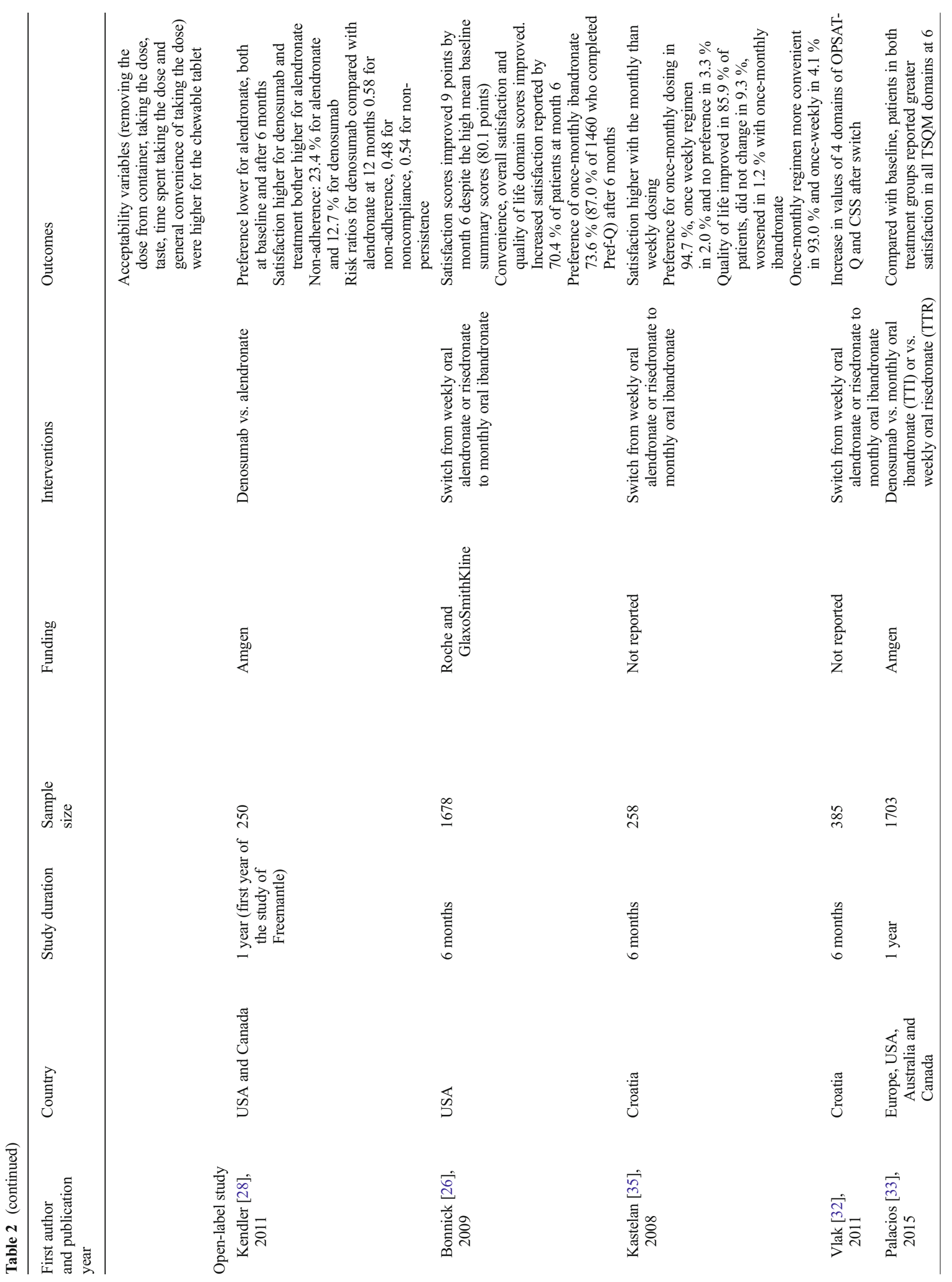


Osteoporosis Patient Satisfaction Questionnaire (OPSATQ) $[26,32]$ and Treatment Satisfaction Questionnaire for Medication (TSQM; consisting of 14 items to assess an individual's perception of effectiveness, side effects, convenience and global satisfaction) [33].

Overall, the crossover studies showed a preference for 6monthly injections or once-monthly oral bisphosphonates over once-weekly oral bisphosphonates $[25,27,29,30]$, or a preference for once-weekly oral bisphosphonates over daily oral bisphosphonates [31,34]. One study compared chewable and sachet calcium/vitamin D supplementation and found a preference for the chewable variant [24]. The sequence of treatments did not influence preferences [27, 30, 31]. Participants considered the lower dosing frequency of oral bisphosphonates to be more convenient [27, 29, 30, 34]. Participants were asked for perceived long-term adherence in 3 studies [25, 31, 34] and thought that the less-frequent dosing regimen would lead to better long-term adherence. Some of the crossover studies also tested for adherence $[24,25,34]$ and found a lower adherence in the less preferred treatment.

The open-label studies included patients who had been on weekly bisphosphonates and who were switched to a monthly regimen $[26,32,35]$ or to another bisphosphonate or denosumab [33] and showed a preference for the less frequently administered treatment. Kendler et al. (2011) found no difference in perceived necessity of osteoporosis treatment between 6-monthly injections or weekly oral bisphosphonates at baseline, but after 6 months, the perceived necessity was higher in the denosumab group. Concern about side effects did not differ between groups. In the study of Kastelan et al. [35], compared to patients who refused to participate in the study $(n=67)$ and those who did not start monthly ibandronate according to the suggestion of their attending doctor $(n=15)$, enrolled patients $(n=258)$ stated less satisfaction with the weekly bisphosphonates at baseline $(p<0.001)$ and were more likely to have adverse events during weekly treatment. Patients reported that the once-monthly dosing regimen better fitted their lifestyle and they preferred the lessfrequent dosing and thought it to be an easier regimen to follow at long term. One study was a randomized clinical trial comparing a weekly oral and a yearly intravenous bisphosphonate, and patients completed the questionnaire without knowing their randomization. The preference for the yearly administered medication was highest [36].

\section{Use of Preferences in Clinical Decision-Making}

Over the last century, the provision and evaluation of medical care saw several paradigm shifts. While medicine relied for ages on experience-based medicine, a shift towards evidencebased medicine followed by evidence-based practice was seen in the twentieth century. While evidence-based medicine and 
practice was largely based on efficacy/effectiveness, safety and increasingly cost-effectiveness, Carr et al. [37] already recognized in 2001 that diseases do not exist in a medical vacuum and that it is impossible to separate disease from an individual's personal and social context. On this line, the twenty-first century is becoming the age of preference-based medicine in which the clinicians become the experts on the medical options, while patients (and their families) bring in their values and preferences. This 'patient centered care' required a whole new area of research into patient-reported outcomes and even more into methods to assess preferences and in approaches to incorporate preferences in the medical office. Preference studies on medication choices, as reviewed above, are valuable to assess which treatments or which treatment attributes are valued (or not) by patients at the group level. Such studies are of great value as they learn that patients' values differ from those of doctors and reveal what aspects of health, treatment or care patients specifically value. Such insights can help when developing treatment guideline (that largely ignores preferences) but can also be an impetus to account for patient needs when developing drugs, also for seemingly futile aspects like treatment administration and frequency of dosing. However, the study by Hiligsmann also emphasized that preferences elicited at the group level show large variance around the estimated coefficients, indicating heterogeneity in preferences between patients. Therefore, in clinical practice, tools are needed that can reveal preferences of individual patients in decisions and support shared decision-making. In osteoporosis, several decision aids are available already, some supporting the patients in the decision on whether or not to start a treatment for (prevention of) osteoporosis, some supporting the decision to choose a specific drug, characterized by its different attributes, and others both aspects of decision [5]. Such decision tools can be either applied in the consultation room by the physician and research nurse or can be used by patients themselves as self-standing online tools. Although a systematic Cochrane review on decision aids showed evidence that decision aids improve knowledge, reduce decisional conflict and succeed in aligning received care with personal values [38], another study revealed that in 1000 office visits in which 3500 medical decisions were made, less than $10 \%$ of decisions met the minimum for informed decision-making [39]. Thus, it appears that even when we really believe in preference-based care, lots of work is still needed [40].

\section{Discussion}

Our review identified several studies that assessed preferences of osteoporotic patients for medications. Different methods have been used including clinical studies and stated-preference methods. In clinical studies, different questionnaires are available to test for preferences and/or convenience. Eliciting preferences seems however not always a straightforward process, as revealed by studies comparing preferences for weekly and monthly oral tablet. Crossover or open-label studies comparing once-monthly and once-weekly oral bisphosphonates revealed a preference for the once-monthly oral tablet $[27,29,30$, $35]$ in patients who had taken both regimens. However, when patients were informed about the limited evidence of efficacy of the once-monthly oral regimen, the preference was in opposite direction with most patients preferring once-weekly oral bisphosphonate with proven efficacy at both the spine and the hip $[22,23]$. In addition, in recent years, an increased use of statedpreference methods like DCEs to elicit preferences in healthcare has been seen [41], which could be interesting to reveal preferences even before receiving a therapy. Formal stated-preference methods use survey/question to elicit patient preferences for hypothetical treatment options in an experimental framework and allow to assess the importance of medication attributes and trade-offs that patients make between them. Alternatively, revealed preference methods are based on observed data related to patients' actual choice. Patients' choice in healthcare however does not often reflect what the patient prefers given imperfect information and revealed preference methods can also not reveal which factors influence the decisions.

One limitation of the design of the clinical studies (especially in open-label studies) is the selection bias. Some of the openlabel studies that switched from a baseline regimen to the study medication included patients with higher rates of dissatisfaction and more side effects at baseline, whereas the patients who were not included did better on the baseline regimen [34]. There is no information available about the preference of the patients who were not included, who did have less side effects and who might have a different preference. Anyhow, in the crossover and open-label studies, the preferred regimen was associated with higher convenience and higher observed adherence. Moreover, patients perceived that their long-term adherence would be better with the preferred regimen. Taking into account the preference of the individual patient would implicate explaining about the different dosing regimens and means of application, the potential benefits of the different regimen and the potential side effects. Prescribing the preferred treatment could improve (long-term) adherence; however, we are lacking evidence of clinical studies.

There are some potential limitations to our review. First, although we reviewed the literature using PubMed, we did not perform a complete systematic review following the PRISMA statement. Since our key words were perhaps limited and we limited our search to one database, we probably missed some papers that however would not have impacted our main findings. Second, we did not provide a quality assessment of the papers. Currently, there are no general guidelines for assessing the quality of stated-preference studies [14•]. Despite these potential limitations, reviewing preferences provide interesting information 
for clinicians and decision-makers which should be aware that osteoporotic patients could reveal preferences for medications and their characteristics. Furthermore, taking into account these preferences when choosing for a treatment for osteoporosis could lead to a higher adherence and compliance. With the increasing importance of the patient perspective and as more studies report on patient preferences, we could also expect that reviews of preferences will be more often made in the future.

\section{Conclusions}

This review revealed that osteoporotic patients report preferences for medications and their characteristics. Although effectiveness and side effects were, not surprisingly, important considerations in treatment preferences, frequency of administration (and in particular less-frequent dosing regimens) is also highly valued by patients, as suggested by Bansback et al. in rheumatic diseases [42•]. Understanding patients' preferences and incorporating them in clinical decision-making could lead to improved osteoporosis care. Patient perceptions and preferences with osteoporosis medications have been shown to impact adherence behaviour [7••] and discontinuation rates [8].

\section{Compliance with Ethics Guidelines}

Conflict of Interest Mickaël Hiligsmann declares the receipt of grants from Amgen, outside the submitted work.

Sandrine P.G. Bours and Annelies Boonen declare no conflicts of interest.

Human and Animal Rights and Informed Consent This article does not contain any studies with human or animal subjects performed by any of the authors.

Open Access This article is distributed under the terms of the Creative Commons Attribution 4.0 International License (http:// creativecommons.org/licenses/by/4.0/), which permits unrestricted use, distribution, and reproduction in any medium, provided you give appropriate credit to the original author(s) and the source, provide a link to the Creative Commons license, and indicate if changes were made.

\section{References}

Papers of particular interest, published recently, have been highlighted as:

- Of importance

•- Of major importance

1. Hernlund E, Svedbom A, Ivergard M, Compston J, Cooper C, Stenmark J, et al. Osteoporosis in the European Union: medical management, epidemiology and economic burden. A report prepared in collaboration with the International Osteoporosis Foundation (IOF) and the European Federation of Pharmaceutical Industry Associations (EFPIA). Arch Osteoporos. 2013;8(1-2):136.
2. Reginster JY. Antifracture efficacy of currently available therapies for postmenopausal osteoporosis. Drugs. 2011;71(1):65-78.

3. Netelenbos JC, Geusens PP, Ypma G, Buijs SJ. Adherence and profile of non-persistence in patients treated for osteoporosis - a large-scale, long-term retrospective study in The Netherlands. Osteoporos Int. 2011;22(5):1537-46.

4. Hiligsmann M, McGowan B, Bennett K, Barry M, Reginster JY. The clinical and economic burden of poor adherence and persistence with osteoporosis medications in Ireland. Value Health. 2012;15(5):604-12.

5. Montori VM, Shah ND, Pencille LJ, Branda ME, Van Houten HK, Swiglo BA, et al. Use of a decision aid to improve treatment decisions in osteoporosis: the osteoporosis choice randomized trial. Am J Med. 2011;124(6):549-56.

6. Brennan PF, Strombom I. Improving health care by understanding patient preferences: the role of computer technology. J Am Med Inform Assoc. 1998;5(3):257-62.

7.• Kendler DL, Macarios D, Lillestol MJ, Moffett A, Satram-Hoang S, Huang J, et al. Influence of patient perceptions and preferences for osteoporosis medication on adherence behavior in the Denosumab Adherence Preference Satisfaction study. Menopause. 2014;21(1): 25-32. Interesting article showing that better perceptions and preferences leads to improved medication adherence in osteoporosis.

8. Barrett-Connor E, Wade SW, Do TP, Satram-Hoang S, Stewart R, Gao G, et al. Treatment satisfaction and persistence among postmenopausal women on osteoporosis medications: 12-month results from POSSIBLE US. Osteoporos Int. 2012;23(2):733-41.

9. Bridges JF. Future challenges for the economic evaluation of healthcare: patient preferences, risk attitudes and beyond. PharmacoEconomics. 2005;23(4):317-21.

10. Strull WM, Lo B, Charles G. Do patients want to participate in medical decision making? JAMA. 1984;252(21):2990-4.

11. Joy SM, Little E, Maruthur NM, Purnell TS, Bridges JF. Patient preferences for the treatment of type 2 diabetes: a scoping review. PharmacoEconomics. 2013;31(10):877-92.

12. Weiss TW, Gold DT, Silverman SL, McHorney CA. An evaluation of patient preferences for osteoporosis medication attributes: results from the PREFER-US study. Curr Med Res Opin. 2006;22(5):94960.

13. Duarte JW, Bolge SC, Sen SS. An evaluation of patients' preferences for osteoporosis medications and their attributes: the PREFER-International study. Clin Ther. 2007;29(3):488-503.

14. Hiligsmann M, van Durme C, Geusens P, Dellaert BG, Dirksen CD, van der Weijden $T$, et al. Nominal group technique to select attributes for discrete choice experiments: an example for drug treatment choice in osteoporosis. Patient Prefer Adherence. 2013;7:1339. Using an innovative method, this article revealed most important anti-osteoporosis medication attributes.

15. Silverman S, Calderon A, Kaw K, Childers TB, Stafford BA, Brynildsen W, et al. Patient weighting of osteoporosis medication attributes across racial and ethnic groups: a study of osteoporosis medication preferences using conjoint analysis. Osteoporos Int. 2013;24(7):2067-77. Interesting study assessing the preferences of patients for medication attributes across different subgroups.

16. Darba J, Restovic G, Kaskens L, Balbona MA, Carbonell A, Cavero P, et al. Patient preferences for osteoporosis in Spain: a discrete choice experiment. Osteoporos Int. 2011;22(6):1947-54.

17. de Bekker-Grob EW, Essink-Bot ML, Meerding WJ, Pols HA, Koes BW, Steyerberg EW. Patients' preferences for osteoporosis drug treatment: a discrete choice experiment. Osteoporos Int. 2008;19(7):1029-37.

18. Fraenkel L, Gulanski B, Wittink D. Patient treatment preferences for osteoporosis. Arthritis Rheum. 2006;55(5):729-35.

19.• Hiligsmann M, Dellaert BG, Dirksen CD, van der Weijden T, Goemaere S, Reginster JY, et al. Patients' preferences for 
osteoporosis drug treatment: a discrete-choice experiment. Arthritis Res Ther. 2014;16(1):R36. Interesting study that included preferences for recent modes of administration using a discretechoice experiment and revealed the substantial heterogeneity in patients' preferences.

20. Ryan M. Discrete choice experiments in health care. BMJ. 2004;328(7436):360-1.

21. Laba TL. Using Discrete Choice Experiment to elicit patient preferences for osteoporosis drug treatments: where to from here? Arthritis Res Ther. 2014;16(2):106. Interesting editorial about the value of a preferences-based study in osteoporosis.

22. Keen R, Jodar E, Iolascon G, Kruse HP, Varbanov A, Mann B, et al. European women's preference for osteoporosis treatment: influence of clinical effectiveness and dosing frequency. Curr Med Res Opin. 2006;22(12):2375-81.

23. Gold DT, Safi W, Trinh H. Patient preference and adherence: comparative US studies between two bisphosphonates, weekly risedronate and monthly ibandronate. Curr Med Res Opin. 2006;22(12):2383-91.

24. den Uyl D, Geusens PP, van Berkum FN, Houben HH, Jebbink MC, Lems WF. Patient preference and acceptability of calcium plus vitamin D3 supplementation: a randomised, open, cross-over trial. Clin Rheumatol. 2010;29(5):465-72.

25. Freemantle N, Satram-Hoang S, Tang ET, Kaur P, Macarios D, Siddhanti S, et al. Final results of the DAPS (Denosumab Adherence Preference Satisfaction) study: a 24-month, randomized, crossover comparison with alendronate in postmenopausal women. Osteoporos Int. 2012;23(1):317-26.

26. Bonnick SL, Silverman S, Tanner SB, Martens M, Bachmann G, Kohles JD, et al. Patient satisfaction in postmenopausal women treated with a weekly bisphosphonate transitioned to oncemonthly ibandronate. J Womens Health (Larchmt). 2009;18(7): 935-43.

27. Emkey R, Koltun W, Beusterien K, Seidman L, Kivitz A, Devas V, et al. Patient preference for once-monthly ibandronate versus onceweekly alendronate in a randomized, open-label, cross-over trial: the Boniva Alendronate Trial in Osteoporosis (BALTO). Curr Med Res Opin. 2005;21(12):1895-903.

28. Kendler DL, McClung MR, Freemantle N, Lillestol M, Moffett $\mathrm{AH}$, Borenstein J, et al. Adherence, preference, and satisfaction of postmenopausal women taking denosumab or alendronate. Osteoporos Int. 2011;22(6):1725-35.

29. Chung YS, Lim SK, Chung HY, Lee IK, Park IH, Kim GS, et al. Comparison of monthly ibandronate versus weekly risedronate in preference, convenience, and bone turnover markers in Korean postmenopausal osteoporotic women. Calcif Tissue Int. 2009;85(5):389-97.

30. Hadji P, Minne H, Pfeifer M, Bourgeois P, Fardellone P, Licata A, et al. Treatment preference for monthly oral ibandronate and weekly oral alendronate in women with postmenopausal osteoporosis: a randomized, crossover study (BALTO II). Joint Bone Spine. 2008;75(3):303-10.

31. Simon JA, Lewiecki EM, Smith ME, Petruschke RA, Wang L, Palmisano JJ. Patient preference for once-weekly alendronate $70 \mathrm{mg}$ versus once-daily alendronate $10 \mathrm{mg}$ : a multicenter, randomized, open-label, crossover study. Clin Ther. 2002;24(11):1871-86.

32. Vlak T, Kastelan D, Lozo P, Aljinovic J, Gradiser M, Mijic S, et al. Monthly or weekly bisphosphonate? Evaluation of satisfaction in patients with postmenopausal osteoporosis using OPSAT-Q questionnaire during the BOOSTER study in Croatia. Clin Rheumatol. 2011;30(12):1549-54.

33. Palacios S, Agodoa I, Bonnick S, Van den Bergh JP, Ferreira I, Ho PR, et al. Treatment satisfaction in postmenopausal women suboptimally adherent to bisphosphonates who transitioned to denosumab compared with risedronate or ibandronate. J Clin Endocrinol Metab. 2015;100(3):E487-92.

34. Kendler D, Kung AW, Fuleihan Gel H, Gonzalez Gonzalez JG, Gaines KA, Verbruggen N, et al. Patients with osteoporosis prefer once weekly to once daily dosing with alendronate. Maturitas. 2004;48(3):243-51.

35. Kastelan D, Lozo P, Stamenkovic D, Miskic B, Vlak T, Kolak Z, et al. Preference for weekly and monthly bisphosphonates among patients with postmenopausal osteoporosis: results from the Croatian PROMO Study. Clin Rheumatol. 2009;28(3):321-6.

36. McClung M, Recker R, Miller P, Fiske D, Minkoff J, Kriegman A, et al. Intravenous zoledronic acid $5 \mathrm{mg}$ in the treatment of postmenopausal women with low bone density previously treated with alendronate. Bone. 2007;41(1):122-8.

37. Higginson IJ, Carr AJ. Measuring quality of life: using quality of life measures in the clinical setting. BMJ. 2001;322(7297):1297300 .

38. Stacey D, Bennett CL, Barry MJ, Col NF, Eden KB, HolmesRovner M, et al. Decision aids for people facing health treatment or screening decisions. Cochrane Database Syst Rev. 2011;10: CD001431.

39. Braddock 3rd CH, Edwards KA, Hasenberg NM, Laidley TL, Levinson W. Informed decision making in outpatient practice: time to get back to basics. JAMA. 1999;282(24):2313-20.

40. Oshima Lee E, Emanuel EJ. Shared decision making to improve care and reduce costs. N Engl J Med. 2013;368(1):6-8.

41. Clark MD, Determann D, Petrou S, Moro D, de Bekker-Grob EW. Discrete choice experiments in health economics: a review of the literature. PharmacoEconomics. 2014;32(9):883-902.

42. Bansback N, Trenaman L, Harrison M. How important is mode of administration in treatments for rheumatic diseases and related conditions? Curr Rheumatol Rep. 2015;17(6):514. Interesting review that discussed the importance of mode of administration in treatments for rheumatic diseases. 